\title{
A primer on hypertension and on the racial / ethnic disparities in diagnosis and management: a comprehensive overview
}

\begin{abstract}
Hypertension is a major cause of premature death worldwide, where it contributes to stroke, cardiovascular and renal disease. Forty percent of adults aged 30-79 years worldwide have hypertension, two-thirds of whom are living in low and middle-income countries. Most adults with hypertension are not fully aware that they have the condition, therefore it often goes ignored and untreated. Of the 1.28 billion people worldwide, who have been reported to have hypertension, data indicates that one in five females and one in four males are included in that estimate. Moreover, data from World Health Organization reports that less than half of adults $(42 \%)$ with hypertension are diagnosed and treated adequately and approximately only one in five adults $(21 \%)$ with hypertension have it under adequate control. One of the worldwide goals for non-communicable diseases is to scale back the prevalence of hypertension by $33 \%$ between 2010 and 2030. In African Americans, readily available thiazide diuretics or Calcium Channel Blockers (CCBs) have been shown to be more effective in lowering blood pressure than Renin Angiotensin System inhibitors (RAS) or $\beta$-adrenergic blockers and are also more effective in reducing cardiovascular disease (CVD) events than RAS inhibitors or adrenergic blockers. The ethnical difference in hypertension and hypertension- related complaint issues are associated with lesser mortality and morbidity pitfalls compared with their white counterparts. These redundant pitfalls from elevated blood pressure have a dramatic effect on life expectancy and career productivity for African American men and women and which is significantly lower than has been reported for Caucasian Americans of either gender. These present challenges remain to be completely understood and give a result to overcome ethnical and racial differences in the frequency and treatment of hypertension. Social determinants of health similar as educational status, access to health care and low income play a crucial part in frequency and blood pressure control rates. Development of appropriate health care programs at the state and public situations to address these issues will be essential to reduce these differences. Thus, the purpose of this paper is to review the prevalence and ethnic disparities in the diagnosis and treatment of hypertension and to suggest steps to improve the outcomes.
\end{abstract}

Volume 5 Issue 6 - 2021

\author{
Ivy Njoloma,' Nasheria Lewis,' Frantz \\ Sainvil, 1,2,3 George P Einstein, 1,3 Andrew \\ Sciranka,' Orien LTulp ${ }^{1,2,3}$ \\ 'University of Science Arts and Technology, Montserrat, BWI, \\ USA \\ ${ }^{2}$ The University of Health and Humanities, British Virgin Islands, \\ BVI, USA \\ ${ }^{3}$ The Einstein Medical Institute, North Palm Beach, FL, USA
}

Correspondence: Ivy Njoloma, University of Science Arts and Technology, Montserrat, BWI, USA, Email njolomai4@gmail.com

Received: November 29, 202| | Published: December 21, 2021

Keywords: hypertension, obesity, population disparities

\section{Introduction}

During any decade of life, for African Americans residing in the United States the prevalence of hypertension is greater than it is for Hispanic Americans, whites, Native Americans, and other subgroups that may be delineated and described by factors of race and ethnicity. ${ }^{1}$ Where treatment control has been deemed adequate the rates of hypertension control have been found to be lower for blacks, Hispanic Americans, and Asian Americans than for whites. Thus, racial and ethnic minority adults have been reported to demonstrate higher rates of hypertension than non-Hispanic white adults, indicating a potentially unhealthy disparity in hypertension control measures and potential successes in therapeutic actions. ${ }^{2}$ Therefore one can see significant differences become more apparent in the prevalence and control rates for blood pressure based on race and ethnicity. The non-Hispanic African Americans have significantly higher rates of hypertension compared to non-Hispanic whites, while Hispanics and non-Hispanic Asians have lower prevalence and control rates than have been observed both groups. The estimates of hypertension prevalence, treatment and control measures among US adults is summarized and depicted in Figure 1 below: Of the hypertensive adults in the US, more than $20 \%$ are recommended to undertake lifestyle modifications while the remaining individuals typically require a combination of lifestyle modifications and medications specific for their individual state of hypertension. Approximately one quarter of those who undergo treatment can achieve acceptable blood pressure control, while the remaining three quarters remain uncontrolled, having either failed or not attempted therapeutic attempts or lifestyle modifications. Of those individuals who choose to or are able undertake effective treatment, blood pressures are likely to reach and maintain normalization, with improvement in the pathophysiologic risks common to untreated individuals.

Thiazide diuretics and Calcium Channel Blockers (CCBs), singly or in combination have been shown to be more effective agents among African Americans in lowering blood pressure than are Renin Angiotensin System (RAS) inhibitors or Beta Blockers and also have been found to be more effective agents for contributing to in reductions in the progression of cardiovascular disease (CVD) events than were RAS inhibitors or adrenergic blockers. The racial and ethnic disparities that have been found in the prevalence and magnitude of hypertension and hypertension-related disease outcomes are associated with higher mortality and morbidity risks in those populations when compared to their white counterparts. The cumulative health risks and unmitigated dangers from chronically elevated blood pressure have a dramatic effect on life expectancy and career productivity for African American men and women which is also significantly lower for Caucasian Americans of either 
gender. These challenges to resolve these disparities are not yet fully understood, and additional measures in the effectiveness and delivery of healthcare must be implemented if we are to overcome the ethnic and racial disparities in the prevalence and treatment of hypertension among disadvantaged groups of the population. Social determinants of healthcare including factors of educational status, greater access to health care and inclusion of lower income likely play key roles in the prevalence of hypertension and effective measures to improve vital sign control rates. Implementation of health care policies at the state and national levels to deal with these issues are likely to become essential elements to scale back these observed disparities in healthcare practices and outcomes. The established criterion for the diagnosis of hypertension is summarized in Figure 2 below and is routinely considered a valid diagnosis if the elevated blood pressure readings are repeated with similar findings on two or more successive days. When determining blood pressure status, care should be taken to obtain the measurements under standardized conditions adhering to the guidelines described in the following paragraphs.

\section{Estimated Hypertension Prevalence, Treatment, and Control Among U.S. Adults'}

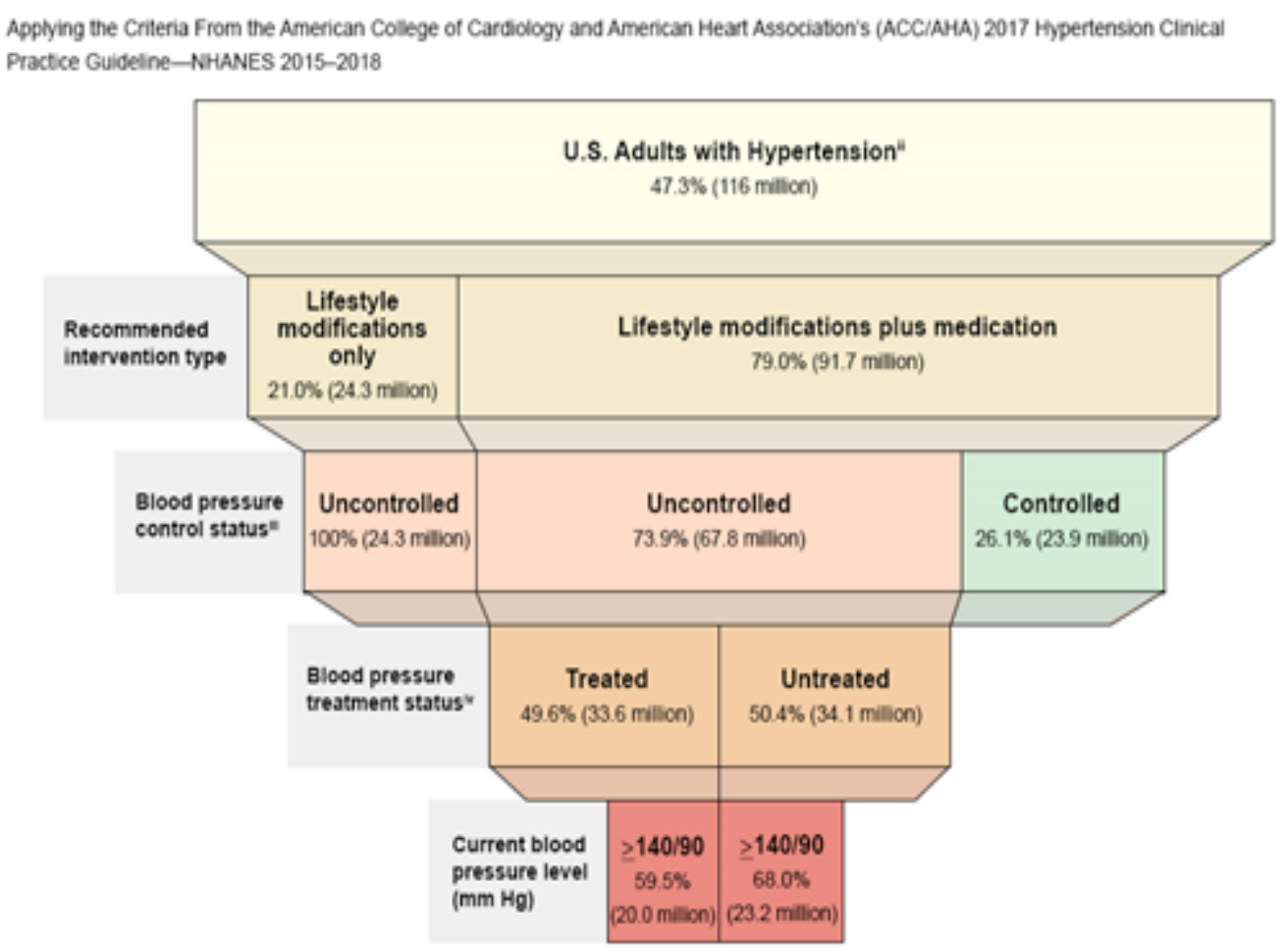

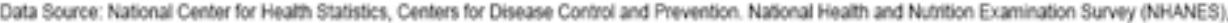

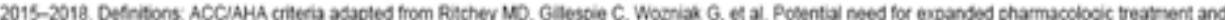

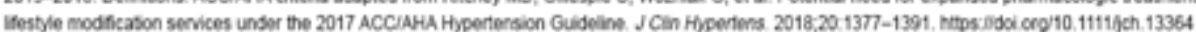

Figure I Summary of estimated hypertension prevalence, treatment and control among US Adults. From NCHS, Centers for Disease Control and Prevention, National Health and Nutrition Survey (NHANES), 2015-2018.

\begin{tabular}{|c|c|c|}
\hline \multicolumn{3}{|c|}{ What is hypertension? } \\
\hline $\begin{array}{l}\text { Blood pressure is the force of circulating } \\
\text { blood against the walls of the body's } \\
\text { arteries, the major blood vessels in the } \\
\text { body. Hypertension is when the force of } \\
\text { the blood pressure is excessive. }\end{array}$ & $\begin{array}{r}140 \\
.90 \\
410\end{array}$ & $\begin{array}{l}\text { Hypertension is } \\
\text { diagnosed if blood } \\
\text { pressure readings are } \\
140-90 \text { or above on }\end{array}$ \\
\hline $\begin{array}{l}\text { Most people with high blood pressure } \\
\text { DO NOT KNOW THEY HAVE IT. }\end{array}$ & & two different days. \\
\hline
\end{tabular}

Figure 2 Diagnosis of Hypertension. From: https://www.who.int/cardiovascular_diseases/hearts/en/.

\section{Obesity is a known contributor to hypertension}

The odds ratio of obesity being a significant contributor to hypertension are greatest in the African American community based in part by factors of age, educational level and socioeconomic status. $^{2}$ The diagnosis of hypertension implies a chronic elevation of blood pressure over an extended duration. As such, uncontrolled hypertension contributes to end-organ vascular damage and is associated with a greater incidence of morbidity and mortality due 
multiple causes including stroke, cardiovascular disease, chronic renal disease and a number of other causes. The average blood pressure of an individual represents the product of cardiac output and systemic vascular resistance on an individual. Patients with arterial hypertension may exhibit an increase in cardiac output, an increase in systemic vascular resistance, or both parameters. The cardiac output in younger age groups is often elevated, while in older patients increased systemic vascular resistance and decreased elasticity of the peripheral and central vasculature contribute to increased stiffness of the vasculature, and the resultant peripheral vascular resistance playa a dominant etiologic pathophysiologic role over time. ${ }^{2}$ As elasticity of the vessels becomes constrained over the course of months or years, the vascular tone may become elevated due to increased $\alpha$-adrenoceptor stimulation or to an increased release of vasoactive peptides including angiotensin or endothelins. ${ }^{2}$ The final consequence is an elevation in cytosolic calcium content in vascular smooth muscle which can facilitate the physiologic processes resulting in vasoconstriction and an inadvertent increase in mean arterial pressure. ${ }^{2}$ Several growth factors, including both angiotensin and endothelin, can also bring about an increase in vascular smooth muscle mass termed vascular remodeling. Both an increase in systemic vascular resistance and an increase in vascular stiffness augment the load imposed on the left ventricle and can induce left ventricular hypertrophy and associated left ventricular diastolic dysfunction. Increased systemic vascular resistance, increased stiffness of the peripheral and central vasculature, and increased vascular responsiveness to the above-mentioned stimuli are central factors in the pathophysiology of hypertension and its consequences. $^{2}$

The morbidity and mortality which follows hypertension result in the development of functional pathophysiologic changes in visceral and other organs. The pathophysiology of hypertension is an investigative area which attempts to explore and clarify the mechanistic causes of hypertension. Hypertension is well recognized as a chronic disease characterized by prolonged elevations of systolic and diastolic elevations in blood pressure and derangements in its regulation during normal physical and emotional activity. Most cases of hypertension may be classified by primary cause as either essential hypertension (also known as primary or idiopathic) or secondary hypertension, where the cause has been found to be due to the consequence of another often treatable contributing factor. About $90-95 \%$ of hypertension cases are typically classified as essential hypertension, where no clear idiopathic cause can be established. Some authorities define essential hypertension as that which has no known explanation, while others may define its cause as a result of disproportionate overconsumption of sodium and under-consumption of potassium, or some combination of both mineral nutrients. Regardless, the actual cause remains unconfirmed, and the etiologic contributors are unknown and far from clear. The two primary types of hypertension are further summarized in Figure 3 depicted below:
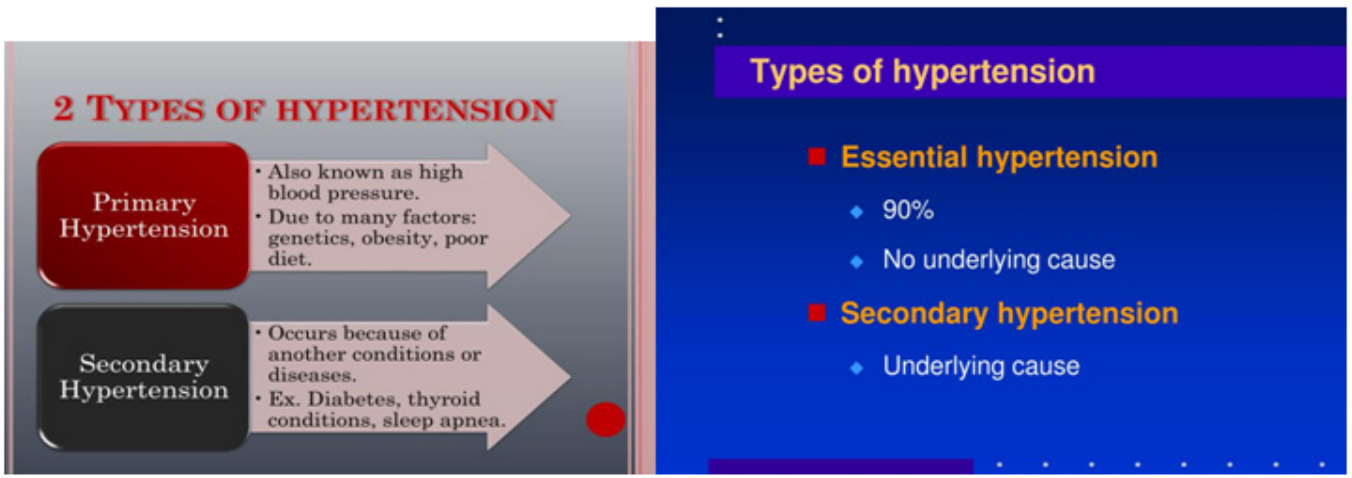

Figure 3 Overview of types of hypertension. From: https://image I.slideserve.com/2968554/types-of-hypertension-l.jpg.

The clinical diagnosis of secondary hypertension affects approximately $10 \%$ of the hypertensive population and indicates that the disorder is likely the result of an identifiable, underlying condition with an established pathophysiologic mechanism, such as a chronic renal disorder, a narrowing of the aorta or kidney arteries, or endocrine disorders like excess secretions and actions of aldosterone, cortisol, catecholamines or other clinically relevant factors. Persistent hypertension of either category is a major risk factor for hypertensive heart disease, coronary artery disease, stroke, aortic aneurysm, peripheral artery disease, and chronic kidney disease. Secondary hypertension therefore can exert broad pathophysiologic changes throughout the body if left untreated as depicted in Figure 4 below: As depicted hypertension of either category can impact tissues throughout the body, often without warning and all too often may remain undected in the early stages of the patophysiologic progression. A summary of affected periphral tissues that may become impacted is depicted in Figure 4 below, and include CNS, vision, Cardiovascular, Renal, and sexual dysfunction including erectile dysfunction (ED) in adult males, often with an onset by middle age:

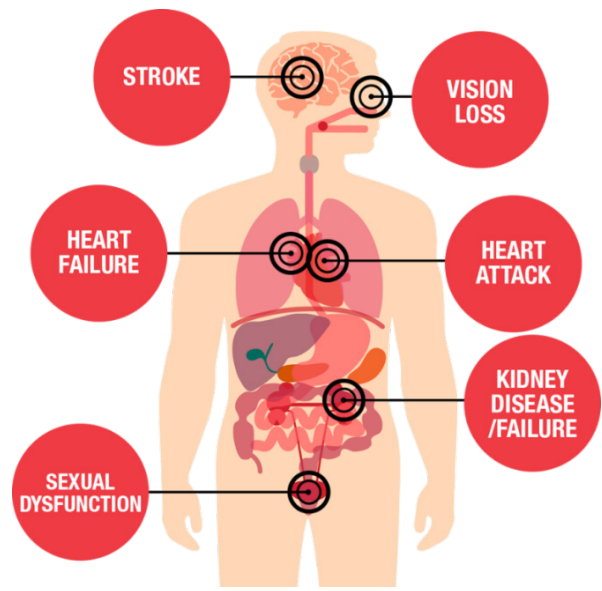

Figure 4 Hypertension affect the whole body. Figure from: https://www. heart.org/-/media/images/health-topics/high-blood-pressure/hbp-threatsillustration-thumbnail.png. 
Blood pressure measurements fall into several categories, depending on the pressures recorded. For an accurate and reliable diagnosis of hypertension, the elevated pressure must have been recorded on two or more occasions under standardized conditions, as numrous factors including nutritional, psychological and a physically relaxed state can impact the blood pressure reading and introduce an artefact that may affect the status. The various categories and levels of systolic and diastolic blood pressure are outlined in Figure 5 below:

\section{Blood Pressure Categories}

\begin{tabular}{|c|}
\hline BLOOD PRESSURE CATEGORY \\
\hline NORMAL \\
ELEVATED \\
\hline $\begin{array}{c}\text { HIGH BLOOD PRESSURE } \\
\text { (HYPERTENSION) STAGE 1 }\end{array}$ \\
\hline $\begin{array}{c}\text { HIGH BLOOD PRESSURE } \\
\text { (HYPERTENSION) STAGE 2 }\end{array}$ \\
\hline $\begin{array}{c}\text { HYPERTENSIVE CRISIS } \\
\text { (consult your doctor immediately) }\end{array}$ \\
\hline
\end{tabular}

SYSTOLIC $\mathrm{mm} \mathrm{Hg}$ (upper number)

\begin{tabular}{|c|}
\hline LESS THAN 120 \\
\hline $120-129$ \\
\hline $130-139$ \\
\hline 140 OR HIGHER \\
\hline HIGHER THAN 180 \\
\hline
\end{tabular}

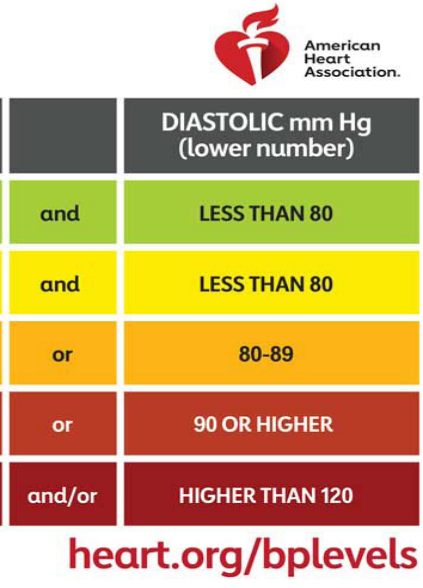

Figure 5A Blood pressure diagnostic categories. From: heart.org/bplevels, American Heart Association. Assumes blood pressure measurements are performed with a standard sphygmomanometer with a blood pressure cuff of an appropriate size for the individual. Pediatric cuffs are not shown. The adult cuff sizes are summarized below:

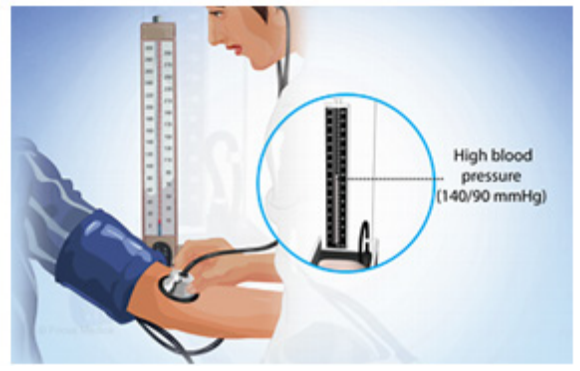

\begin{tabular}{|l|l|l|}
\hline \multicolumn{2}{|l|}{ Arm Circumference } & Recommended Cuff Size (width $\mathrm{x}$ length in cm) \\
\hline $\mathrm{cm}$ & in & \\
\hline $22-26$ & $8.7-10.2$ & $12 \times 22$ (small adult) \\
\hline $27-34$ & $10.6-13.4$ & $16 \times 30$ (adult) \\
\hline $35-44$ & $13.8-17.3$ & $16 \times 36$ (large adult) \\
\hline $45-52$ & $17.7-20.5$ & $16 \times 42$ (extra-large adult) \\
\hline
\end{tabular}

Figure 5B Diagnostics tests for hypertension: accurate measurement of blood pressure.

To determine an accurate blood pressure, the medical professional or assistant places an inflatable arm cuff around the arm usually just above the elbow, inflates the cuff sufficiently that no sounds can be heard, and then applies a pressure-measuring gauge as depicted above in combination with the sphygmomanometer and stethoscope, or listening device. The blood pressure readings are traditionally recorded in millimeters of mercury $(\mathrm{mm} \mathrm{Hg})$ and have has two numbers. The first, or upper, number measures the pressure in your arteries when your heart beats (systolic pressure) and is the first sound the health professional or other attendant can discern as the graded level of inflation of the cuff is relieved. The second, or lower, number measures the pressure in your arteries between beats (diastolic pressure) and represents that pressure when the cuff is sufficiently deflated such that no more heart sounds can be discerned by the attendant. ("Prehypertension Disease Reference Guide - Drugs. com") "According to the American Heart Association, your blood pressure is normal if it is in the range of $120 / 80 \mathrm{~mm} \mathrm{Hg}$ or modestly lower." ("Elevated blood pressure - Diagnosis and treatment - Mayo ...") A patient is usually seated for up to 5 minutes before reliable measurements can be made under standardized conditions. The position of the patent (i.e., sitting, prone, supine, etc.) and time in minutes since last period of exercise can often dramatically affect the readings. As outlined by the American heart Association and others, simple tips to obtain the most accurate and reproducible BP readings include:
I. Patient is sitting comfortably, with arm resting comfortably on a table or similar surface and at a level of the mid abdomen, and with an appropriate size cuff placed on upper arm approximate 1-2 inches above the crease in the elbow: a too small cuff size can introduce an error of 2-10 $\mathrm{mm} \mathrm{Hg}$., and cuff over wrapping due to use of a cuff that is too large for the patient's arm can introduce an artifact of $5-50 \mathrm{~mm} \mathrm{Hg}$;

II. The patient should not engage in conversation during the measurement, as the conversation can be not only distracting to the observer talking measurements as the needs a quiet environment to hear the heart sounds clearly during the measurement process and when lacking can introduce an artefact of up to $10 \mathrm{~mm} \mathrm{Hg}$.;

III. Always take the BP when the patient has an empty bladder and has no gastrointestinal comfort. A full bladder or gastrointestinal distress can introduce an artifact of up to 10 $\mathrm{mm} \mathrm{Hg}$;

IV. The patient should be sitting comfortably with legs uncrossed. Crossing legs during the measurement can introduce an artefact of $2-4 \mathrm{~mm} \mathrm{Hg}$;

V. The patient seating environment should have both a back and foot supports, such as in a chair with feet comfortably reaching the floor. Improper seating can introduce an artefact of up to 6 $\mathrm{mm} \mathrm{Hg}$. 
Thus, technical failure to abide by the above guidelines may introduce significant artefacts in a patient's true blood pressure, and potentially contribute to an inaccurate diagnosis and proposed treatment plan for the patient.

${ }^{6}$ BP guidelines. https://targetbp.org/wp-content/uploads/2017/11/ Measuring Blood Pressure In-Office-pdf.jpg and https://support. omadahealth.com/hc/article_attachments/360035978753/Cuff circumference_chart.png

When evaluating a patient for primary hypertension the protocol typically includes several important laboratory tests, which are regularly available at your local hospital or medical clinic or physician's or other provider's office. The specific laboratory tests include but are not limited to the following:

I. Fasting blood glucose (FBS or FBG); If FBG is elevated one may wish to add an oral glucose tolerance or a 2-hour post prandial blood glucose measurement;

II. Complete / full blood count (CBC or FBC);

III. Lipid profile, including triglycerides, LDL, HDL, and total cholesterol concentrations;

IV. Serum creatinine with estimated glomerular filtration rate (GFR) adjusted for race as indicated, in addition to serum sodium, potassium, and calcium concentrations;

V. Thyroid stimulating hormone (TSH);

VI. Urinalysis, to include a screening test for glucose (glycosuria) and proteins (proteinuria);

VII. Optional Testing may include an Echocardiogram, Uric acid, and Urine albumin to creatinine ratio

VIII. Nutritional factors, including clinical signs of macronutrient, micronutrient, trace element nutrition in addition to potential toxic effects of both essential and non-essential elements.
Screening for new onset of uncontrolled secondary hypertension may include assessment for the following considerations:

Q: Does the patient have new-onset or uncontrolled hypertension? If so, some related conditions to consider include:

I. Drug-resistant/induced hypertension

II. Abrupt onset of hypertension

III. Onset of hypertension at $<30$ years of age

IV. Exacerbation of previously controlled hypertension

V. Disproportionate target organ damage for the degree of hypertension present

VI. Accelerated/malignant hypertension

VII. Onset of diastolic hypertension in those $\geq 65$ years of age

VIII. Unprovoked or excessive hypokalemia or magnesium deficiency.

${ }^{10}$ From Whelton PK et al. J Am Coll Cardiol 2018;71: e127-e248

\section{The racial and ethnic subgroup in hypertension prevalence and new ACC/AHA high blood pressure management guidelines}

The age-adjusted death rates by race and ethnic origin are depicted in Figure 6 below. As depicted here, the racial disparity has been recognized for decades in hypertension and hypertension-related outcomes, with African Americans experiencing greater risks than age matched Caucasians. The blood pressure levels measured have been consistently higher among African Americans, who also experience an earlier onset of the hypertension. ${ }^{9}$ The higher blood pressure levels for African Americans are associated with higher rates of stroke, endstage renal disease and congestive heart failure. ${ }^{9}$ despite the similarity in awareness and treatment levels of high blood pressure between the two population groups, evidence for racial differences in hypertension control rates are evident. ${ }^{9}$

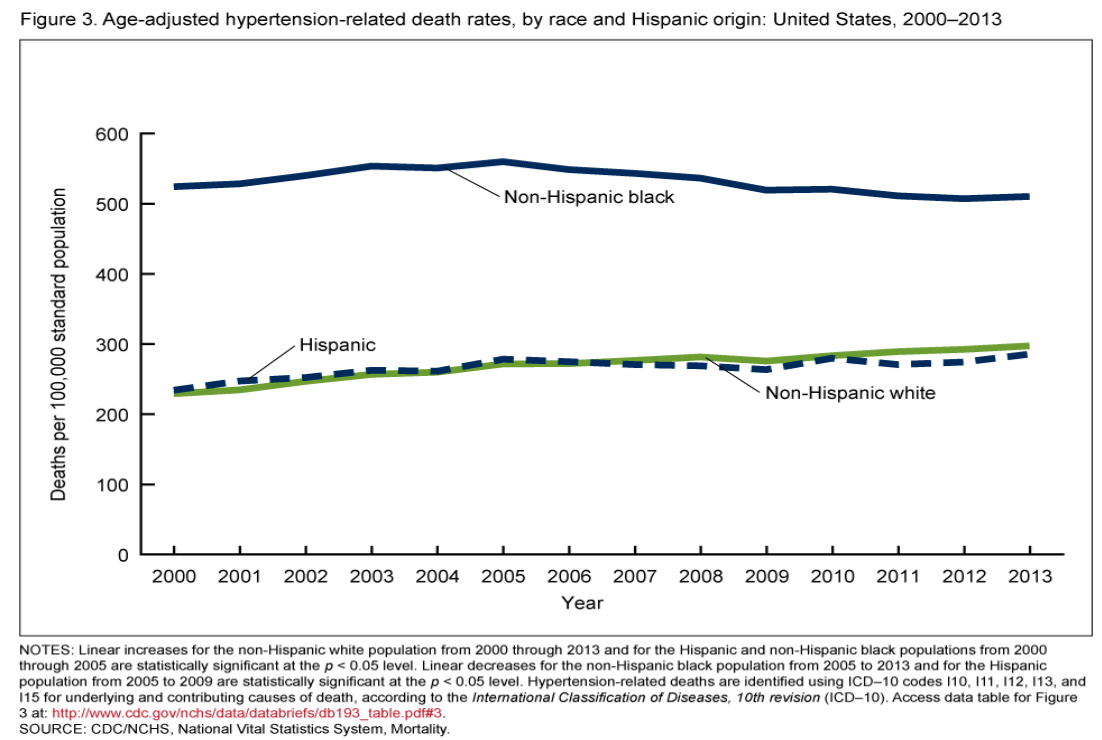

Figure 6 Age adjusted death rates by race and ethnic origin, Figure 3 from National Vital Statistics System: Mortality. CDC/NCHS.

In Figure 7, the data indicates that African Americans in the United States, at any decade of their life, have a statistically greater prevalence of hypertension than that which has been shown for
Hispanic Americans, whites, Native Americans, and other subgroups when defined by race and ethnicity. In addition, hypertension control is less successful for African Americans, whose rates reflect lower 
rates than Hispanic Americans, Asian Americans and for whites of corresponding ages. Gender differences are also apparent, as among men with hypertension, non-Hispanic white adults had a higher prevalence of controlled high blood pressure $(53.8 \%)$ than did nonHispanic black (43.8\%), non-Hispanic Asian (39.9\%), and Hispanic $(43.5 \%)$ adults. For women, hypertension, among non-Hispanic white adults demonstrated controlled hypertension (59.1\%). In non-Hispanic blacks hypertension rates were higher (52.3\%) than in non-Hispanic Asian (46.8\%) adults. In Hispanic Americans, the lower control rates were found to have been attributed to lack of awareness, inadequate, not appropriate or non-treatment, whereas in African Americans, awareness and treatment efforts were at least as high as in whites, but the magnitude of hypertension was more severe, and some therapeutic agents were less effective in achieving at BP control. ${ }^{10}$

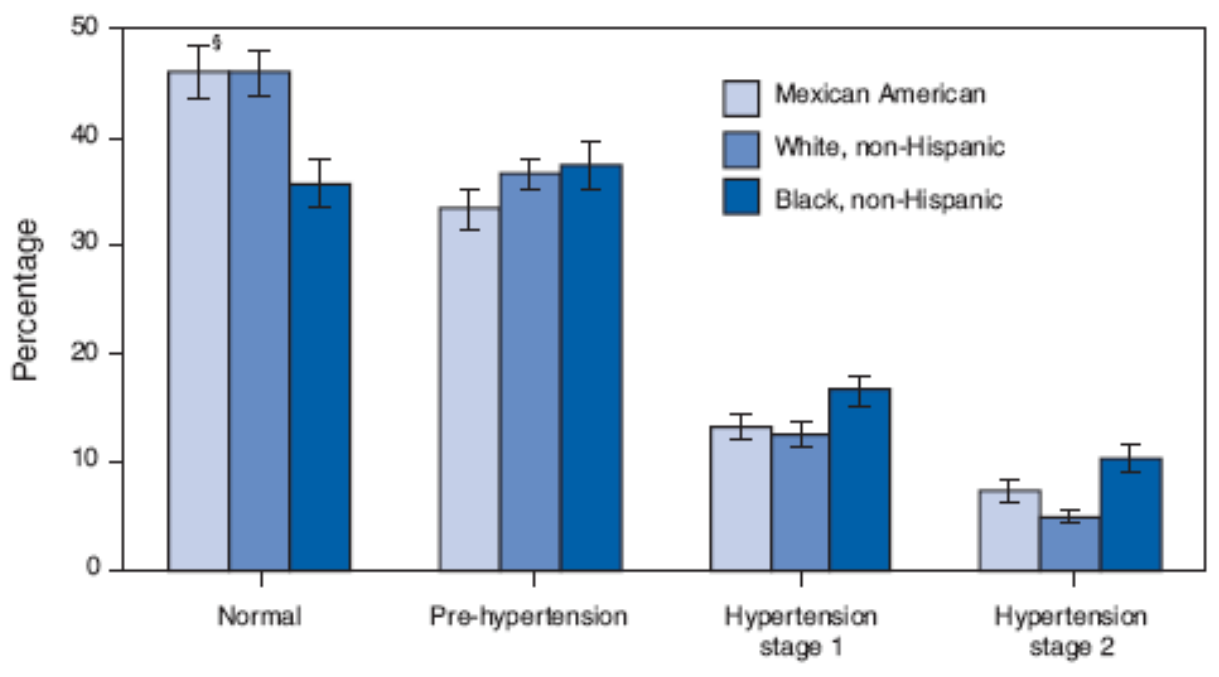

Blood pressure category

Figure 7 Prevalence of stages of hypertension in Mexican Americans, Whites, and Non-Hispanic African Americans. From: https://www.cdc.gov/mmwr/preview/ mmwrhtml/figures/m624qsf.gif.

Morbidity and mortality attributed to hypertension are also more common in African Americans and Hispanic Americans than occurs in whites. African Americans have a 1.3-fold greater risk of nonfatal stroke, 1.8-fold greater risk of fatal strokes, 1.5 -fold greater risk of Heart Failure, and 4.2-fold greater risk of end stage renal disease (ESRD). ${ }^{10}$ Hispanic Americans have lower rates of hypertension awareness and treatment than those of whites and Black people, as well as a higher prevalence of comorbid CVD risk factors including obesity and diabetes mellitus (DM). The distribution of the underlying causes of hypertension related death in the United States during the ten years spanning 2003 to 2013 is depicted in Figure 8 below:

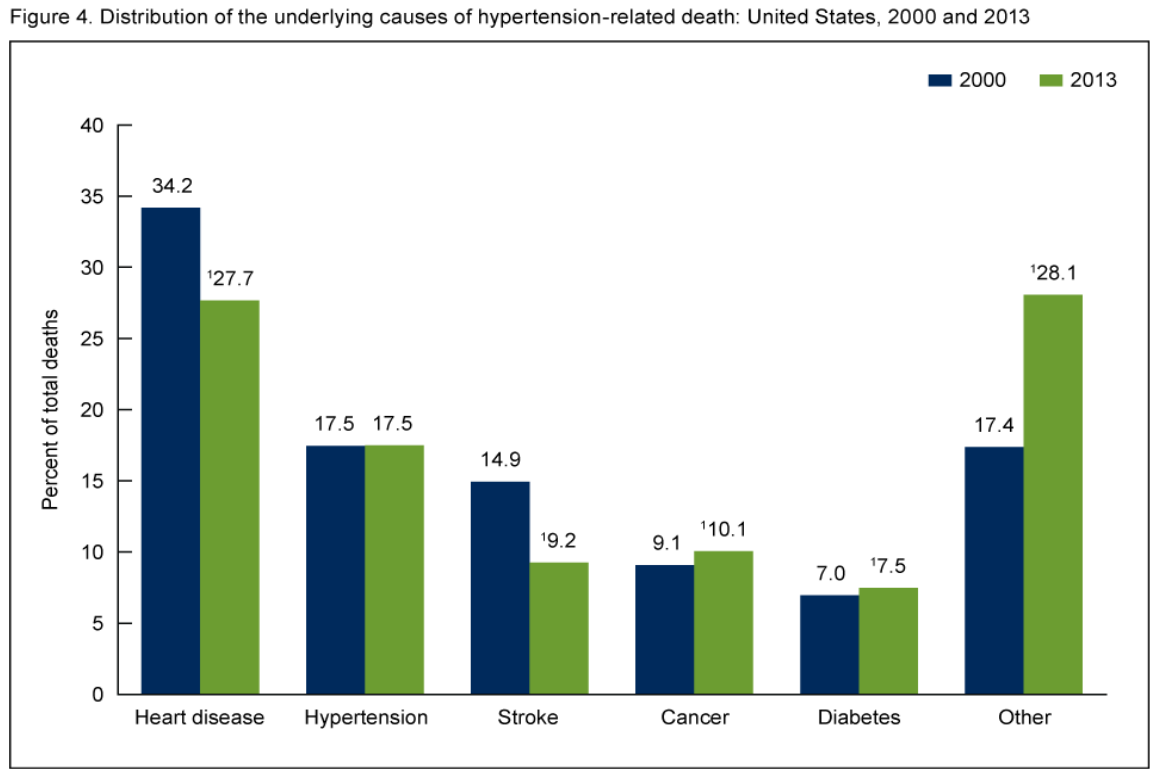

'Significantly different from year 2000 at the $p<0.01$ level. NOTE: The causes were coded for Heart disease as 100-109 and 120-151, Hypertension as 110-115, Cancer as C00-C97, Stroke as 160-169, and Diabetes as E10-E14, based on the International Classification of Diseases, 10 th revision
SOURCE: CDC/NCHS, National Vital Statistics System, Mortality.

Figure 8 From Figure 4 of CDC/NCHS. National Statistics System: Mortality. 
In 2014, age-adjusted hypertension-attributable mortality rates per 1000 persons for non-Hispanic white, non-Hispanic black, and Hispanic-American men and women were 19.3 and 15.8, 50.1 and
35.6, and 19.1 and 14.6, respectively an as reported in 2015-2016 depicted in Figure 9 below:

Figure 4. Age-adjusted prevalence of controlled hypertension among adults with hypertension aged 18 and over, by sex and race and Hispanic origin: United States, 2015-2016

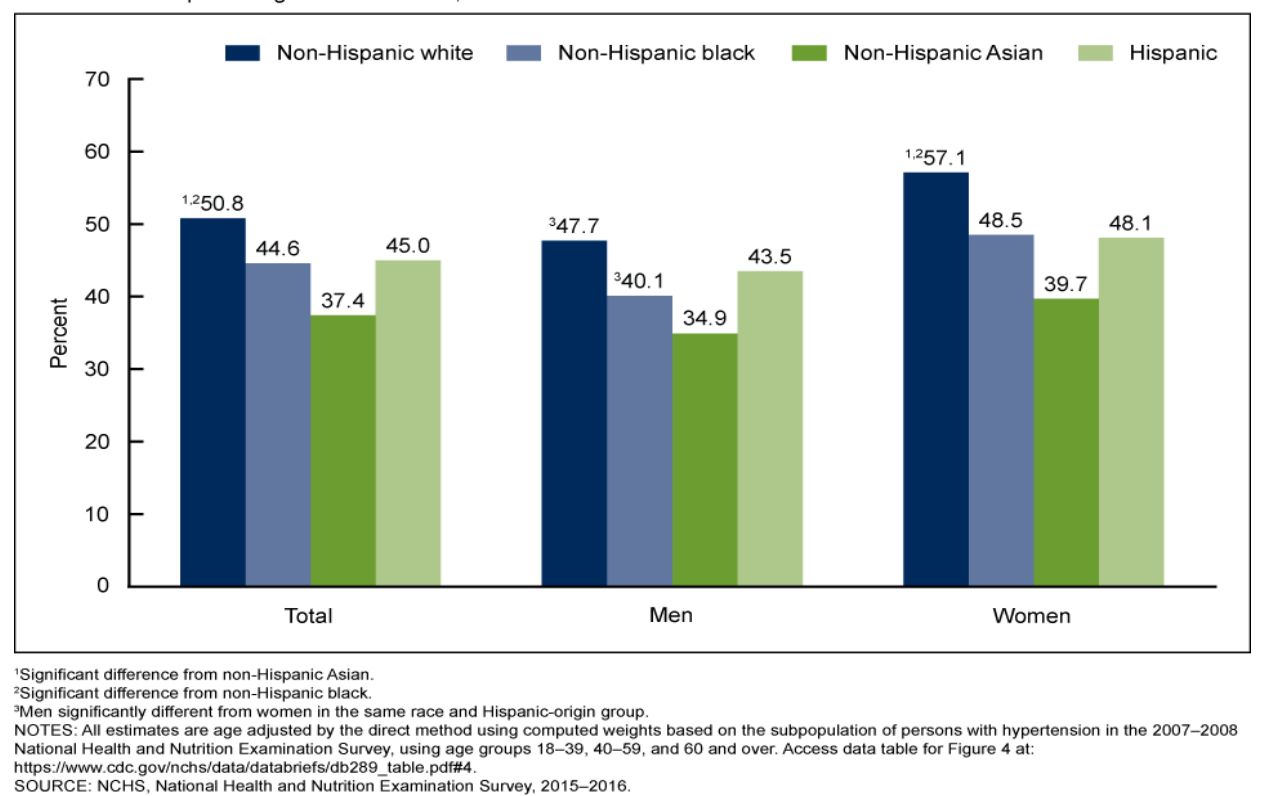

Figure 9 From Figure 4 of CDC/NCHS. National Statistics System: Mortality.

As reflected in Figure 10 below, hypertension significantly increases the mortality rates among African Americans. The data indicate that African-Americans are prone to develop high blood pressure at younger ages than other ethnic and racial groups in the U.S. and thus are at greater risk of common pathophysiologic complications which follow later in life. The extent that this increase may be linked to magnesium or other nutritional deficiencies or factors that can influence alterations in vascular smooth muscle contractility or relaxation has not been determined, but micronutrient supplementation including magnesium has been reported to be beneficial in some populations..$^{14,15}$

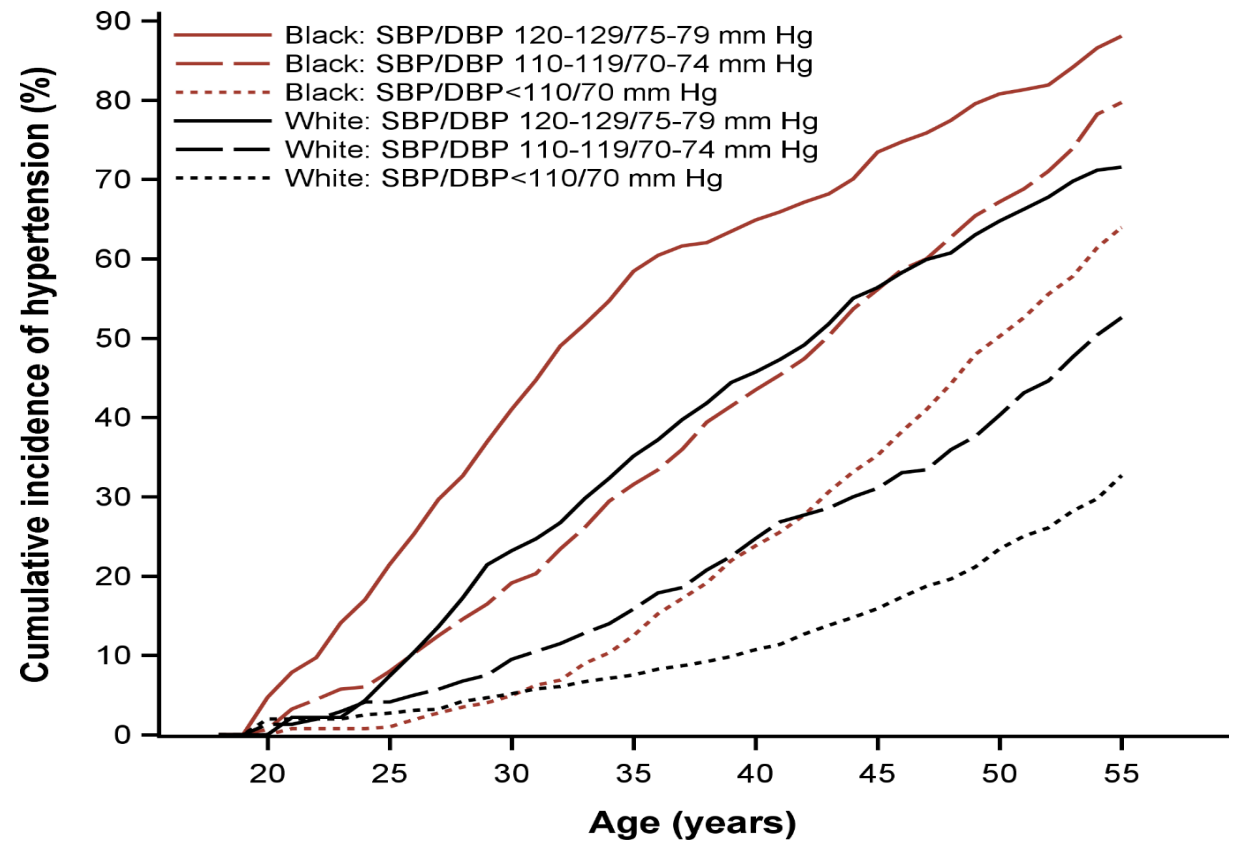

Figure 10 Age of onset of hypertension among African Americans and White Americans. From: https://www.ahajournals.org/cms/asset/4I6a203e- I7f7-4ce4846I-I8bd I f8cc978/jah33322-fig-0002.png. 
As depicted in Figure 11 below, African-Americans are more likely to develop complications associated with high blood pressure such as stro ke, kidney disease, blindness, dementia, and heart disease. Hypertension, Diabetes, and Stroke are depicted below: African Americans are illustrated in the brown bars, and whites in the light blue bars ad graphically illustrate the differences in prevalence with advancing age in each category.
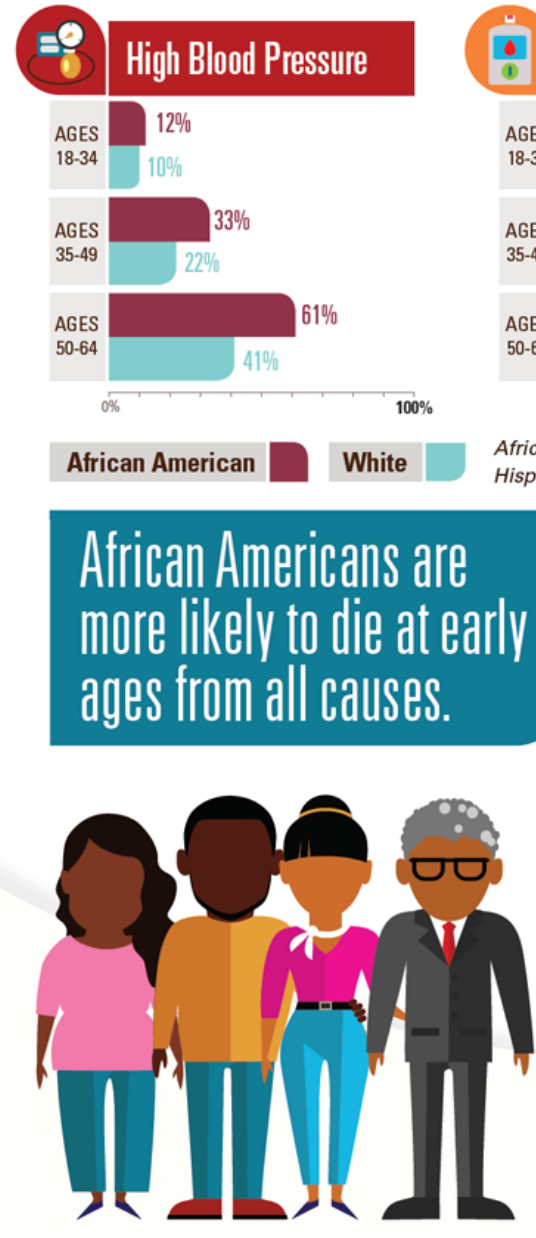

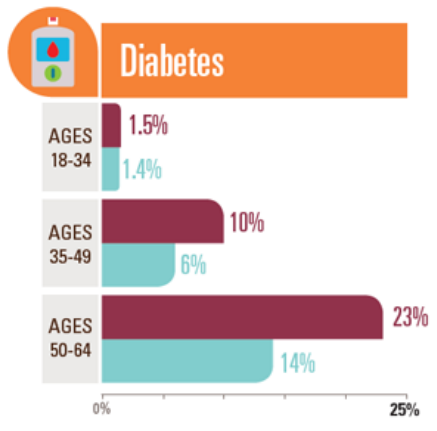

African Americans and whites include Hispanic and non-Hispanic origin.

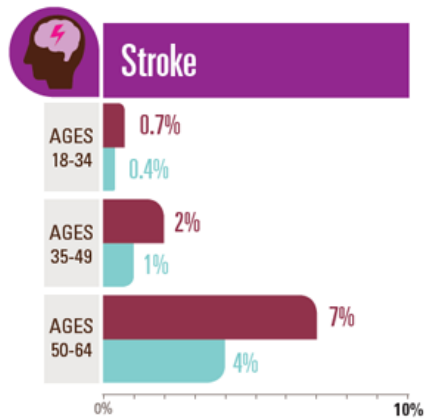

SOURCE: Behavioral Risk Factor Surveillance System, 2015.

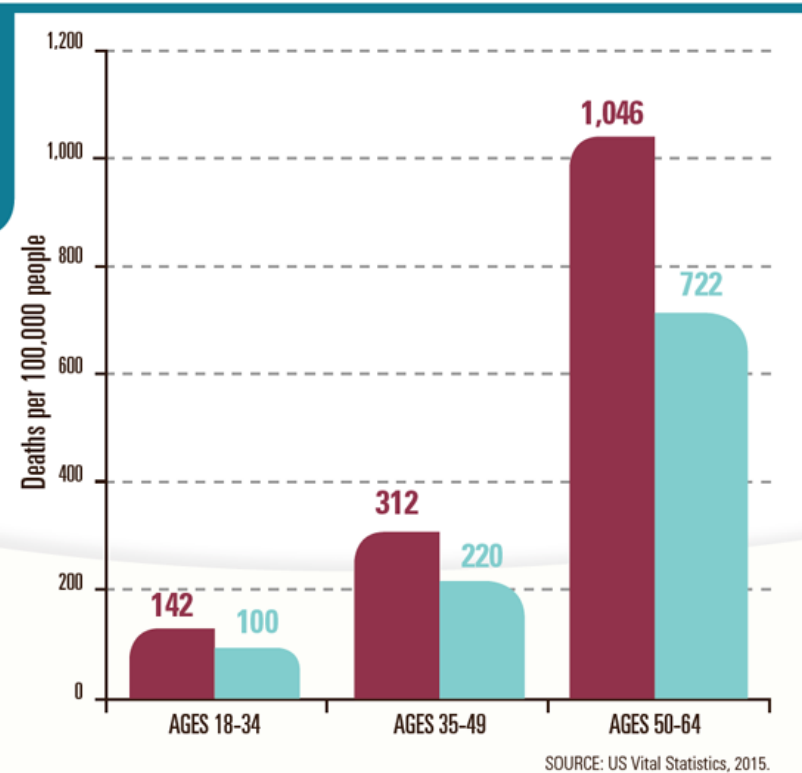

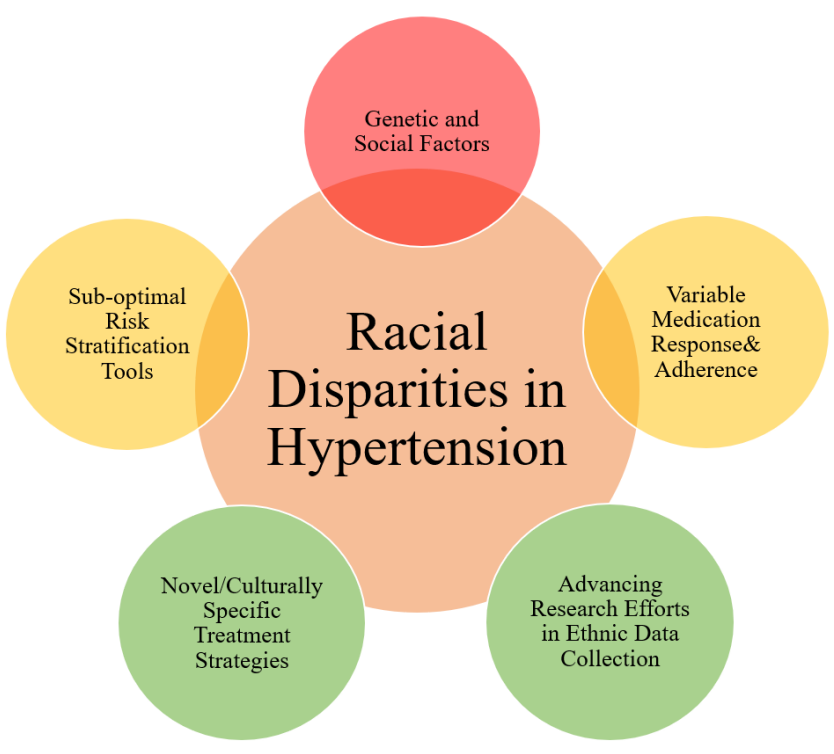

Figure I I Central Illustration: Racial Disparities in Hypertension. Major factors (red), reasons for worse outcomes (yellow) and future directions for improving disparities in ethnic minorities (green). ${ }^{12}$

Citation: Njoloma I, Lewis N, Sainvil F, et al.A primer on hypertension and on the racial / ethnic disparities in diagnosis and management: a comprehensive overview. Int J Fam Commun Med. 202I;5(6):229-239. DOI: I0.15406/ijfcm.202I.05.00250 
Some researchers believed that high blood pressure in African Americans may be triggered by the following factors:

\section{Genetic and physiologic factors}

I. High rates of high blood pressure in African Americans may be partially due to the genetic make-up of people of African descent.

II. Researchers have uncovered some facts: In the U.S, blacks respond differently to high blood pressure drugs than do other groups of people.

III. Black people in the U.S. also seem to be more sensitive to salt, which increases the risk of developing high blood pressure, and which plays a role in designing their treatment plans.

\section{Environmental factors}

I. Some scientists support the concept that high blood pressure in African Americans is due to factors unique to the experience of Black people in the U.S.

II. Black Americans: $41 \%$ of Black people have high blood pressure, as compared to $27 \%$ of whites. In addition, black people in the U.S are more likely to be overweight than blacks in other countries, implicating nutritional factors and/or hormonal factors, or combinations of the two.
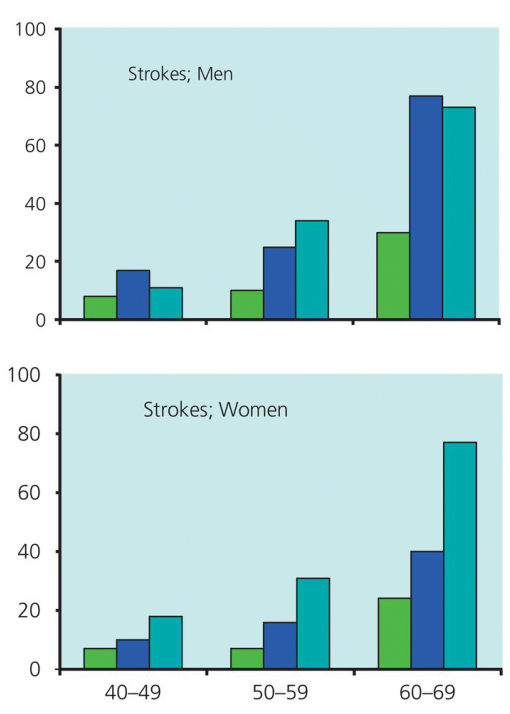

III. Some experts think that social and economic factors including discrimination and economic inequality are at least in part responsible for this difference.

IV. A range of the primary contributing factors proposed are summarized in Figure 11 below:

The detailed reasons for the racial disparities in elevated blood pressure and hypertension-related outcomes risk among African Americans and other populations remain unclear. The racial disparity in hypertension and hypertension-related outcomes has been apparent for decades with African American populations consistently experiencing greater risks than Caucasians as mentioned earlier. ${ }^{9}$ However, the clinical pathophysiological implications resulting from the disparities of hypertension for prevention and clinical management are substantial. African American men and women with greater hypertension risk and warranting interventions have been focused on these differences. In addition, focused research to identify the factors attributed to those disparities in risk burden is essential to gain insight in how best to address the gaps. ${ }^{9}$ The racial disparities in hypertension and hypertension-related disease outcomes have related mortality and morbidity risks when compared to their white counterparts. ${ }^{9}$ The excess risks from sustained elevated blood pressure have a dramatic effect on lowering life expectancy for African American men and women, and which is significantly less than for Caucasian Americans for stroke and heart disease in both males and females as depicted in Figure 12 below:
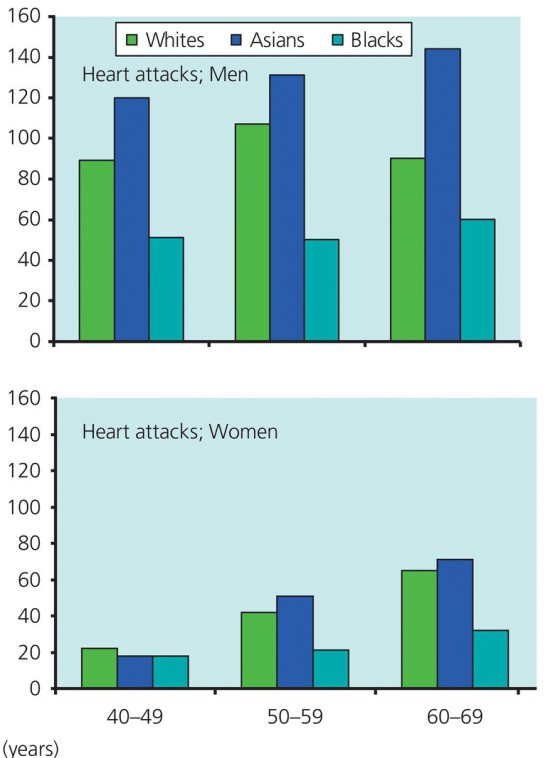

Figure 12 Effect of age, gender and ethnicity on mean incidence of stroke and cardiac ailments. From: https://www.cdc.gov/mmwr/preview/mmwrhtml/figures/ m624qsf.gif.

This figure clearly illustrates the relative prevalence of stroke and heart conditions by age categories from age 40 to 69 in people of white, Asian and African American ethnicity. In both genders, African Americans have a greater incidence of stoke ailments than all other groups from age 50, while for heart ailments, the incidence in greater for Asians in most age categories.

\section{Hypertension management and guidelines}

Lifestyle modification including such measures as weight reduction and control, dietary modification, and graded increases in physical activity are important in both African Americans and Hispanic
Americans as a first line approach for prevention programs and/or adjunctive therapy for hypertension. However, the recommendations for adoption of changing one's lifestyle habits is often a challenging proposition in some ethnic minority patients because of poor social support, limited access to exercise facilities, unclear fitness goals, availability of healthier foods, and financial considerations all of which may be needed to effect the changes. Thus, the greater prevalence of lower socioeconomic status may impede access to the basic living necessities needed to bring about change including improved medical care, availability of necessary medications, access to convenient fitness resources and nutritional factors. Consideration 
must also be given to learning new styles and preference, addressing personal beliefs, values, and culture from a positive perspective which vary substantially among diverse cultural, minority and ethnic groups occurring concurrently within the population.

Medications are highly useful components of a treatment plan and may be best individualized for each patient to achieve the greatest compliance and therapeutic success, as medications that may prove effective in one patient may not necessarily be equally effective in others seeking the same outcomes. The basic principles of antihypertensive drug selection for ethnic minorities varies from those of non-Hispanic Black people. In African Americans thiazidetype diuretics and CCBs have proven to be more effective in lowering blood pressure when given as monotherapy or as initial agents in multidrug regimens. In addition, thiazide-type agents are superior to drugs that inhibit the RAS (ACE inhibitors, ARBs, renin inhibitors, and beta blockers) for prevention of selected clinical outcomes in African Americans. In African Americans, use of thiazide diuretics or CCBs have been shown to be more effective in lowering BP than were RAS inhibitors or beta blockers and also were more effective in reducing CVD events than were RAS inhibitors or alpha blockers.
The RAS inhibitors were recommended in African Americans patients with hypertension, DM, and nephropathy, but they failed to offer an advantage over diuretics or CCBs in hypertensive patients with DM without nephropathy or HF. ${ }^{10}$

The four most common drug classes examined (thiazide diuretic, $\mathrm{CCB}$, ACE inhibitor, or ARB) were effective in lowering blood pressure and in improving cardiovascular or renal outcomes when taken as prescribed. Thus, except for the combination of ACE inhibitors and ARB regimens or containing a combination of these drug classes are deemed reasonable agents to achieve the target blood pressure control. ${ }^{10}$ Furthermore, the combination of an ACE inhibitor or ARB with a $\mathrm{CCB}$ or thiazide diuretic was shown to produce similar blood pressure lowering effects in African Americans as with other racial or ethnic groups. For African Americans who are unable to achieve adequate control with three drugs, sometimes termed as resistant hypertension, a common treatment paradigm which addresses this dilemma is summarized in the following algorithm (Figure 13). This algorithm reflects a roadmap to consider when proposing a treatment plan for individual patients of any racial or ethnic group.

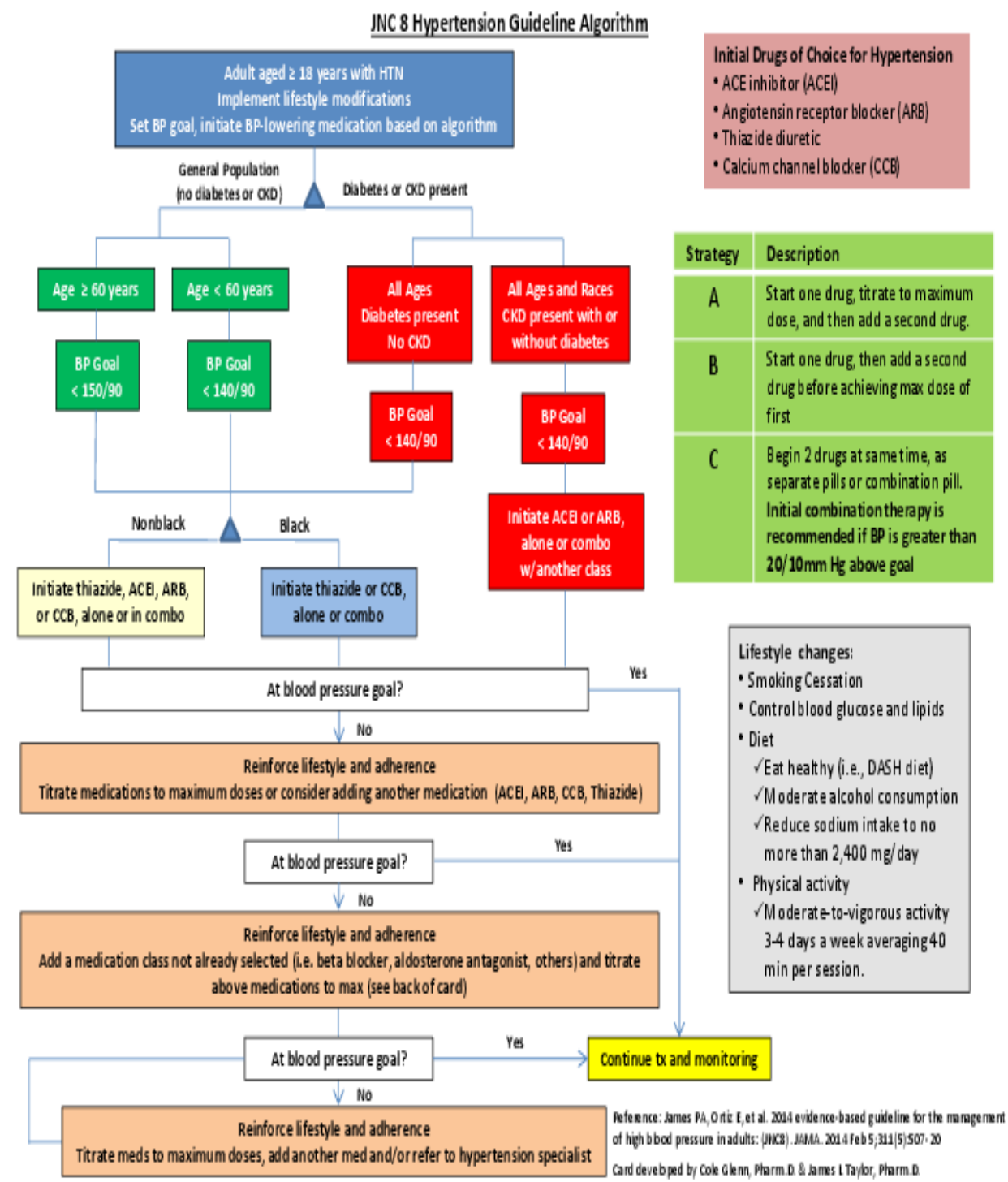

Figure 13 An algorithm guideline for the diagnosis and treatment of hypertension. From: James, PA et al. JAMA 20I4: 3 I I (5) 507-520. 


\section{Conclusion}

Hypertension is a major contributing cause of premature death worldwide. Persistent hypertension is a major risk factor for hypertensive heart disease, coronary artery disease, stroke, aortic aneurysm, peripheral artery disease, and chronic kidney disease. The racial disparity in hypertension and hypertension-related outcomes has been recognized for decades with African Americans demonstrating greater risks and less successful treatment than Caucasians. Blood pressure levels have consistently been higher for African Americans with greater odds ratio and an earlier onset of hypertension. While awareness programs and treatment levels of high blood pressure have been similar, racial differences in control rates are evident. Significant challenges remain to better understand and overcome the ethnic and racial disparities that are apparent in the prevalence and treatment of hypertension among different racial and ethnic groups, and their variations with age and between genders. Established social determinants including differences in individual health, educational and socioeconomic status, access to health care, nutritional status and low family income play a key role contributing to the prevalence and blood pressure control rates among different populations. The implementation of health care policies at the State and National levels to address these issues will be essential to reduce these disparities and improve the outcomes of attempts for intervention. While genetic and environmental factors that contribute to hypertension are incompletely understood, the evolution of precision tailored medicine and development of more refined Arteriosclerotic Vascular Disease (ASCVD) risk tools for Asians and Hispanics will help to optimize appropriate risk-adjusted blood pressure management and treatment and improve the health, well-being and productivity of the relevant populations. $^{12}$

\section{Acknowledgments}

The authors want to thank the numerous faculties of the University of Science Arts and Technology, Montserrat with special recognition to Professors Bruce H. Robinson and Andrew Sciranka for their brilliant lectures and discussions on this topic.

\section{Conflicts of interest}

The author declares there is no conflict of interest.

\section{References}

1. WHO. 2017.

2. Foëx P, Sear JW. Hypertension: pathophysiology and treatment. Continuing Education in anesthesia critical care \& pain. 2004;4(3):71-75.

3. Tulp OL, Awan AR, Lewis N, et al. Can epigenetic expression contribute to the development of the obese phenotype? Adv Obese Weight Manag \& Control. 2021:11(3)98-101.

4. Foëx P, Sear JW. Hypertension: pathophysiology and treatment. Continuing. 2004;4(3):71-75.

5. AHA. 2021

6. AMA. 2017.

7. Whelton PK. Testing for primary hypertension and Secondary hypertension. J Am Coll Cardiol. 2018;71:e127-e248.

8. https://support.omadahealth.com/hc/article_attachments/360035978753/ Cuff_circumference_chart.png

9. Lackland DT. Racial differences in hypertension: implications for high blood pressure management. Am J Med Sci. 2014;348:135-38.

10. Paul K Whelton, Robert M Carey, Wilbert S Aronow, et al. ACC/AHA/ AAPA/ABC/ACPM/AGS/APhA/ASH/ASPC/NMA/PCNA Guideline for the Prevention, Detection, Evaluation, and Management of High Blood Pressure in Adults: A Report of the American College of Cardiology/ American Heart Association Task Force on Clinical Practice Guidelines. Hypertension. 2018;71:e13-e115.

11. CDC. 2021

12. Saeed A, Dixon DL, Yang E. Racial Disparities in Hypertension Prevalence and Management: A Crisis Control? Apr 06, 2020, Journal American College Cardiology. 2020165(18):2098-2104.

13. AHA. 2021

14. Tulp OL, Bell A, Einstein GP, et al. Can Traditional Chinese Medicine Formulas Treat BMI-associated Hypertension Effectively? Submitted. Advances in Obesity and Weight Regulation and Management. 2021;11(3):72-74.

15. Sainvil F. Anatomy and Physiology. In: KH Saladin editor. $8^{\text {th }}$ Ed, McGraw-Hill Publishing. 2018. 